\title{
Transferencia de la entonación y bilingüismo: el caso de San Antonio, Texas ${ }^{1}$
}

\author{
Transfer of intonation and bilingualism: \\ the case of San Antonio, Texas
}

\author{
JOSEFA DORTA \\ Universidad de La Laguna, Departamento de Filología Española. \\ SEGAI Laboratorio de Fonética. España. Correo electrónico: jdorta@ull.edu.es
}

El objetivo de este trabajo es analizar la entonación del español hablado en San Antonio, Texas. El análisis se ha centrado en declarativas e interrogativas neutras extraídas de un corpus semi-espontáneo de informantes norteamericanos hablantes de español por herencia familiar. Nuestra hipótesis es que la entonación del español de San Antonio tiene gran influencia del mexicano dada su situación geográfica y la cantidad de población mexicana que reside en esta poblada ciudad y sus alrededores. Los resultados evidencian coincidencias con la entonación mexicana, como el marcado ascenso tonal final de las interrogativas, pero también diferencias que podrían estar motivadas por la influencia del inglés.

Palabras clave: entonación, habla semi-espontánea, español de San Antonio, Texas, declarativas e interrogativas.

The aim of this paper is to analyze the intonation of the Spanish spoken in San Antonio, Texas. The analysis is focused on neutral declarative and interrogative sentences drawn from a semi-spontaneous corpus obtained from North American native speakers who speak Spanish (heritage speakers). The first hypothesis is that the Spanish intonation in San Antonio has a great influence of Mexican due to its geographical location and the Mexican population residing therein. The results show similarities with the Mexican intonation, as marking the strong end tonal rise of interrogative sentences, but also differences that might be caused by the influence of English language.

Key words: intonation, semi-spontaneous speech, Spanish in San Antonio, Texas, declarative and interrogative sentences.

\footnotetext{
${ }^{1}$ Este trabajo se ha realizado en el marco del proyecto de investigación Estudio comparativo de la entonación y del acento en zonas fronterizas del español (FFI2014-52716-P), financiado por el Programa Estatal de Fomento de la Investigación Científica y Técnica de Excelencia, Ministerio de Economía y Competitividad de España.
} 


\section{INTRODUCCIÓN}

La Ciudad de San Antonio se encuentra al sur del estado de Texas en el condado de Béxar. El censo de 2010 de EE.UU. registraba una población de 1.327 .407 personas de las que 838.952 eran de origen hispano (Ennis et al. 2010); en 2013 había aumentado esta población a 1.256 .102 personas $(54,9 \%)$ frente a los 796.962 anglos. La cercanía con México, entre otras razones históricas, ha determinado que en la actualidad más del 50\% de los pobladores de San Antonio sea de origen mexicano, aunque el inglés prevalece frente al español.

El estudio de esta última lengua se ha centrado en EE. UU., en general, y en San Antonio, en particular, en el nivel léxico y segmental descuidando, por tanto, el prosódico y, más concretamente, el de la entonación, a pesar de que esta es fundamental para entender tanto las similitudes y diferencias interlingüísticas como las existentes entre variedades de una misma lengua pues, como afirmaba Juan Manuel Sosa hace años, "uno de los factores más característicos y resaltantes que permite inmediatamente a un hablante identificar el origen geográfico de su interlocutor es precisamente la entonación" (1999: 177). La presencia de los dos idiomas en San Antonio -español e inglés- supone considerar la condición social y lingüística de uno y otro. El inglés, según Moreno Fernández (2008: 200), condiciona el perfil de las comunidades en que se utiliza el español, interfiere en sus rasgos lingüísticos, afecta a su presencia pública y tercia sobre las actitudes lingüísticas de los hispanohablantes. Pero el contacto entre ambas lenguas puede interferir también en la entonación (Zubizarreta y Nava 2010) puesto que sus características varían de una lengua a otra (Ladd 1996); por tanto, el intercambio inadecuado de patrones entonativos puede tener repercusiones en la interpretación del mensaje (Kelm 1995) o en la recepción del discurso por parte del oyente (Anderson-Hsieh et al. 1992). Por todo ello, si ya sabemos que los hablantes españoles de primera generación y los hablantes que heredan el español de sus familiares incorporan términos ingleses a su vocabulario, es relevante considerar, por una parte, hasta qué punto interfiere el inglés en la entonación de los informantes que hablan español en San Antonio, Texas y, por otra, dada la fuerte presión de lo mexicano, saber si adoptan los esquemas entonativos del español de México, ya sea porque es la lengua que aprendieron de pequeños de sus padres o abuelos o porque la estudian por motivos diversos.

\section{Marco teórico}

La Geolingüistica o Geografía Lingüistica es una disciplina que cuenta con un desarrollo importante en los últimos ańos. Su objetivo es, como sabemos, describir la extensión y disposición en el espacio de los hechos de las lenguas y de sus variedades; para ello ha diseñado su propia metodología de investigación y su manera de presentar los rendimientos obtenidos en los llamados atlas lingüisticos. Los importantes avances en el seno de la misma traspasan hoy los límites nacionales para vincularse a un ámbito internacional 
muy ambicioso. Es el caso del Atlas Multimedia de Prosodia del Espacio Románico (AMPER), cuyo objetivo es dar cuenta, por primera vez, de la prosodia de las lenguas románicas y de sus variedades. Uno de los ámbitos lingüísticos estudiados en AMPER es el español y sus variedades, tanto de Espańa como de América. En el presente estudio nos ocupamos del español de un ámbito excepcional, San Antonio, en la frontera EE. UU.-México, donde la presencia de lo mexicano (habitantes, cultura, variedad lingüística, etc.) es un referente para los texanos (Marcos Marín 2014; Manzanares Rivera 2015), pero, al mismo tiempo, el inglés es la lengua oficial. Por tanto, estamos ante una situación de lenguas en contacto español/inglés, inglés/español (Silva Corvalán 1996; Lacorte and Leeman 2009; Escobar and Wölck 2009; Marcos Marín 2006) donde la presencia y diversidad de la población hispana -en Texas y en general en EE. UU- trae como consecuencia que las variedades lingüísticas del español confirmen la procedencia geográfica y social de los hablantes.

El desconocimiento de la entonación del español de San Antonio ha determinado que nos hayamos planteado como objetivo hacer una aproximación a su estudio. La hipótesis de partida es que la entonación del español hablado en la ciudad y en su entorno tiene una gran influencia de la variedad mexicana por la situación geográfica de esta zona, por la cantidad de población mexicana que reside en ella y por la transmisión generacional de la lengua. Para poder comparar la entonación de los informantes de San Antonio con la mexicana, es preciso conocer cuáles son los rasgos más importantes que definen a esta última. El problema fundamental es que, a pesar de la extensión de México y de las variedades de español en ese amplio territorio, los estudios se han centrado fundamentalmente en Ciudad de México (Martín Butragueño 2006: 105) por lo que todavía hay un gran desconocimiento de la entonación mexicana.

El pretonema de las declarativas del mexicano, según Sosa, se caracteriza como en otras variedades del espańol: el punto más alto se sitúa "invariablemente en el primer pico siguiendo a la primera sílaba acentuada, luego de la cual se inicia el descenso" (1999: 195), por lo que se produce un escalonamiento descendente. En cuanto al tonema, este mismo autor encuentra, incluso en el habla neutra, un movimiento final circunflejo $\left(\mathrm{L}+\mathrm{H}^{*}\right.$ L\%) que origina "la ruptura de la línea descendente hacia la línea de base tonal" (191); este final circunflejo no es causado aparentemente por motivaciones pragmáticas, "sino por características dialectales sistemáticas, aunque nuestros datos no nos permiten ser categóricos con respecto a esta materia" (197).

Con anterioridad, otros autores habían señalado que la configuración tonemática circunfleja es característica del habla popular de México (Matluck 1965), mientras que Quilis (1985) decía que en hablantes universitarios, si bien se da el patrón del español general, esto es, "el fundamental desciende gradualmente hasta el final del enunciado" (161), el contorno circunflejo es el más común; esta terminación ha sido encontrada también por Martín Butragueño (2004) en enunciados declarativos emitidos por hombres y mujeres de un nivel sociocultural bajo procedentes de Ciudad de México.

Más recientemente, Mota et al. (2010) afirman que el patrón nuclear en la variedad de español mexicano de Distrito Federal (hoy Ciudad de México) es el más común del 
español ( $\left.\mathrm{L}^{*} \mathrm{~L} \%\right)$, pero encuentran también los finales circunflejos $\left(\mathrm{L}+\mathrm{H}^{*} \mathrm{~L} \%\right)$, tanto en declarativas de foco ancho como estrecho. Congosto (2012), por su parte, siguiendo la metodología de AMPER, analiza la entonación del español en un corpus formal de una hablante bilingüe nacida en El Paso, Texas, en la frontera entre Estados Unidos y México, y concluye lo siguiente: las declarativas se caracterizan por un primer pico tonal alineado con la tónica en los sintagmas con final agudo y pospuesto en los finales llanos y esdrújulos; a partir del pico, "se inicia la declinación que termina con un tonema final en cadencia" (151). De estos datos se concluye, por tanto, que al menos en este estilo de habla formal no se da el final circunflejo señalado por otros autores para el habla mexicana.

En lo que respecta a las interrogativas absolutas del español mexicano, Sosa (1999: 126, 127, 200, 202) señala que una de sus características es que la altura tonal de todo el enunciado es más elevada que la de las declarativas y, al contrario que en estas, el escalonamiento pretonemático es ascendente a partir del tono inicial $\mathrm{H} \%$. El tonema, por su parte, presenta la subida a partir del núcleo, por lo que la sílaba nuclear es baja $\left(\mathrm{L}^{*}\right)$ y se coloca en la línea de base tonal. A partir de la acentuada, la F0 experimenta un ascenso muy amplio con un tonema $\mathrm{L}^{*}+\mathrm{H} \mathrm{H} \%$; ello hace que esta variedad se asemeje al castellano pues, según Quilis, las interrogativas absolutas se caracterizan "por un final ascendente del fundamental, que arranca, generalmente, del final de la última sílaba tónica, si el final del enunciado es paroxítono o proparoxítono; si es oxítono, empieza en la penúltima o en la última sílaba” (1993: 429). No obstante, tanto Sosa (1999) como Quilis (1985) coinciden en señalar que el ascenso de los finales mexicanos es mayor que el de las interrogativas castellanas por lo que estas, según el primer autor, pueden representarse con $\mathrm{L}^{*} \mathrm{H} \%$. Además del tonema descrito, Quilis encuentra "ocasionalmente" en sus materiales de México un final circunflejo como el que registra en Puerto Rico y Gran Canaria (1993: 471). Ávila (2003), por su parte, analizó 140 construcciones interrogativas extraidas de conversaciones libres de informantes de Ciudad de México. En relación con las absolutas, la autora señala que el contorno tipico final es ascendente $\left(L+H^{*} H \%\right)$, aunque encuentra también un tonema descendente ( $\left.L^{*} L \%\right)$. El ascendente, además, presenta dos alotonemas o variantes en distribución complementaria: $\mathrm{L}+\mathrm{H}^{*} \mathrm{H} \%$ aparece en las emisiones con final paroxítono -en los que el ascenso empieza a definirse en la sílaba nuclear y se eleva en la posnuclear o en la siguiente- y $\mathrm{H}^{*} \mathrm{H} \%$ en los oxítonos; en cambio, ambas variantes aparecen en finales proparoxítonos o esdrújulos. En el estudio, ya citado, de Mota et al. (2010), las interrogativas absolutas terminan generalmente en ascenso de modo que en preguntas del tipo ¿̇tiene mermelada? encuentran $\mathrm{L}^{*}+\mathrm{H}$ en el acento inicial y L* LH\% en el final; en este último caso, según los autores, la tónica alcanza un mínimo que continúa en la postónica, elevándose luego la F0 bruscamente. Por último, en el estudio de Congosto (2012) se indica que las interrogativas comienzan con tono ascendente que culmina en un primer pico; este se pospone siempre alineándose con la postónica. La configuración tonemática es siempre ascendente con la particularidad de que los valores de F0 más bajos se dan en los enunciados con final agudo y los más altos en los enunciados con final esdrújulo. Además, "se corrobora que este ascenso parte de un punto cercano a la línea de base tonal, por debajo de la media del enunciado" (2012: 155). 


\section{Marco Metodológico}

El corpus de análisis del presente trabajo fue obtenido en 2012 en la ciudad de San Antonio mediante entrevistas directas en una situación cómoda para los hablantes. Analizamos declarativas e interrogativas extraídas de un corpus semi-espontáneo obtenido mediante el planteamiento de situaciones de la vida cotidiana con el objetivo de recoger respuestas habituales en ese tipo de situaciones. Véanse los ejemplos siguientes:

(1) Situación: Vas por la calle y encuentras a un niño que no lleva reloj; este te pregunta: "Señor ¿̨me puede decir la hora?” ¿Cómo contestarías normalmente en esta situación?

Una de las respuestas obtenidas es la ilustrada en la figura 1; se puede apreciar en la curva de F0 que se superpone al espectrograma que el final es descendente.

Figura 1. Son las doce con quince minutos (hombre AOTx6) ${ }^{2}$

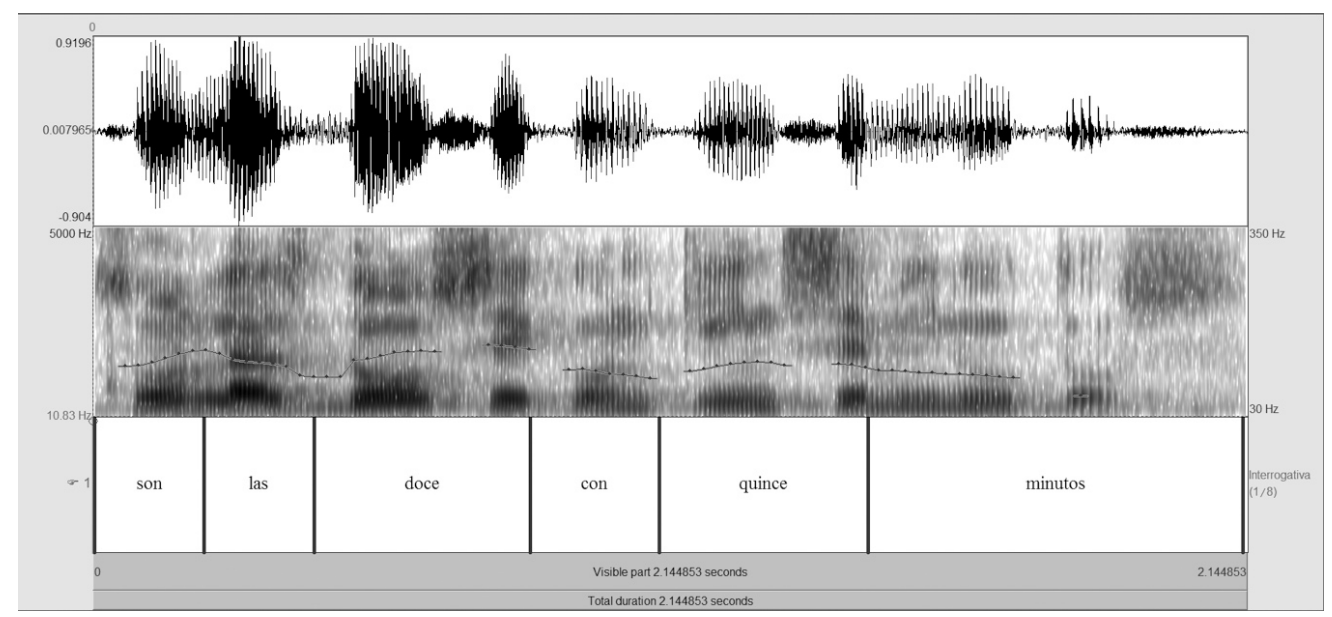

(2) Situación: Ves a un señor mayor que intenta cruzar la calle pero tiene problemas. Si intentas ayudarlo ¿qué le dirías?

Véase en la figura 2 una de las respuestas que hemos registrado; la F0 al final es ascendente.

\footnotetext{
${ }^{2}$ La ilustración ha sido realizada con Praat, versión 6.0.19.
} 
Figura 2. ¿Usted necesita ayuda? (hombre AOTx8)

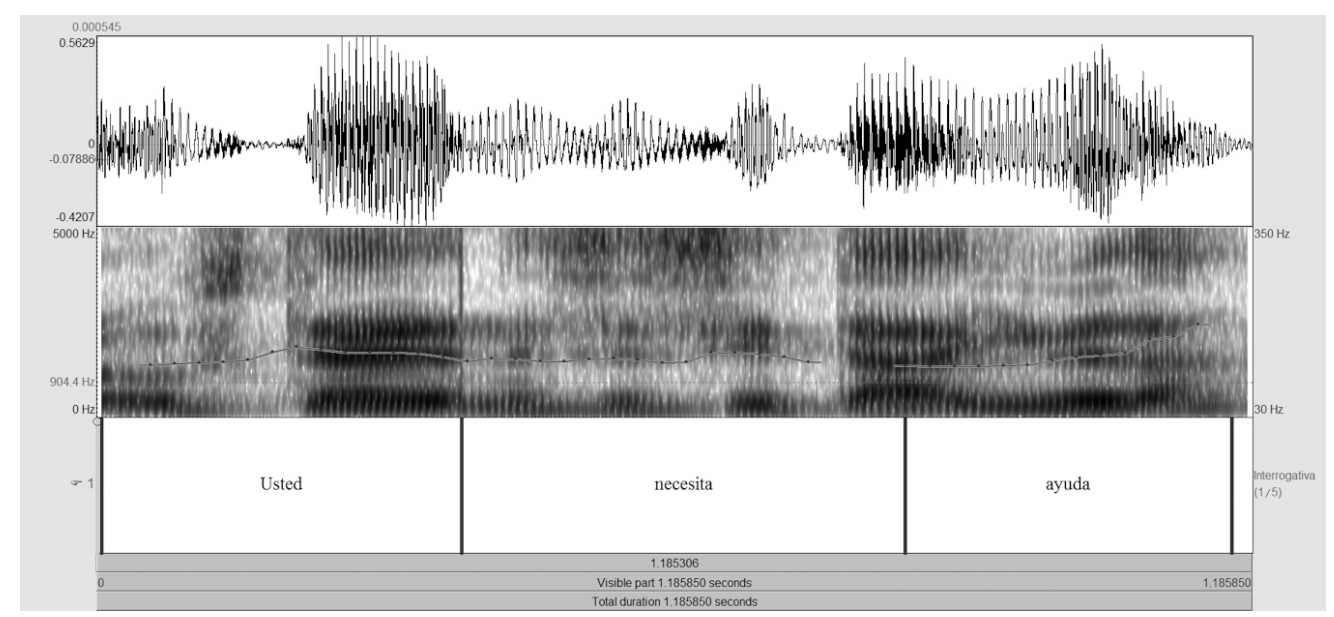

El corpus analizado fue emitido por cinco informantes, tres hombres (AOTx6, de 50 años; AOTx8, de 30 años y AOTx10, de 25 ańos) y dos mujeres (AOTx 5 y AOTx7, de 36 y 25 ańos, respectivamente) ${ }^{3}$ con estudios universitarios. Los tres hombres nacieron en San Antonio y en ellos se advierte claramente la influencia del mexicano debido a sus antecedentes familiares. En AOTx6 dicha influencia es más evidente que en AOTx8 y AOTx10 en los que tiene más peso el inglés; en los registros más espontáneos de estos dos últimos, como la conversación, alternan ambas lenguas. En lo que respecta a las mujeres, hablan muy bien el español con acento mexicano: AOTx5 nació en Laredo, aunque se trasladó desde muy pequeña a San Antonio; recibió su educación en lengua inglesa puesto que en su ciudad no había educación bilingüe, pero sus padres y abuelos -residentes en Nuevo Laredo, ciudad del lado mexicano- le hablaban en español. Por su parte, AOTx7 nació en San Antonio y es hija de madre mexicana (Ciudad de México); además, en San Antonio vive en un núcleo con fuerte presencia hispana, sobre todo mexicanos.

Las frases analizadas son 150: 86 declarativas neutras y 54 interrogativas absolutas no pronominales. En las dos modalidades hemos registrado frases con inicio tónica-átona (en adelante TA) y frases que comienzan por átona-tónica-átona (ATA); en cuanto a los finales, hemos visto asimismo dos tipos: final agudo o átono-tónico (AT) y final llano o átono-tónico-átono (ATA).

El corpus fue grabado con una grabadora portátil profesional Zoom H4n y la señal fue tratada con el programa Goldwave (versión 4.25) para eliminar los ruidos y optimizarla con una frecuencia de muestreo de $16 \mathrm{Khz}$. El análisis acústico se llevó a cabo con rutinas

\footnotetext{
${ }^{3}$ En la identificación de los informantes: A (español de América), O (Estados Unidos), Tx (Texas), número impar (mujer) y número par (hombre).
} 
creadas ad hoc en el entorno Matlab (versión 7.0.4.) ${ }^{4}$ y el etiquetado de las frases se hizo con el sistema Sp_ToBI partiendo de una propuesta de Dorta (2013) que en este trabajo se modifica en parte. La propuesta de 2013 aparece en las tablas 1 y 2.

Tablas 1 y 2. Representación de las variantes e invariantes y esquema acústico de los acentos tonales (tabla 1, izquierda) y acentos de frontera final (tabla 2, derecha) (adaptación de Dorta 2013: 72, 76).

\begin{tabular}{|c|c|c|}
\hline ESTRUCTURA PROFUNDA & ESTRUCTURA SUPERFICIAL & NIVEL ACÚSTICO \\
\hline Invariante fonológica & Variantes & Esquema del contorno tonal \\
\hline \multirow{2}{*}{$/ \mathrm{L}^{*}+\mathrm{H} /$} & {$\left[\mathrm{L}^{*}+\mathrm{H}\right]$} & \\
\hline & {$\left[\mathrm{L}^{*}+! \mathrm{H}\right]$} & \\
\hline \multirow{2}{*}{$/ \mathrm{L}+\mathrm{H}^{*} /$} & {$\left[\mathrm{L}+\mathrm{H}^{*}\right]$} & \\
\hline & {$\left[\mathrm{L}+>\mathrm{H}^{*}\right]$} & \\
\hline \multirow{2}{*}{$/ \mathbf{L}^{* /}$} & [L*] & \\
\hline & {$\left[! \mathrm{H}+\mathrm{L}^{*}\right]$} & \\
\hline \multirow{5}{*}{$/ \mathrm{H}^{* /}$} & {$\left[\mathrm{H}^{*}\right]$} & \\
\hline & {$\left[i \mathrm{H}^{*}\right]$} & \\
\hline & {$\left[! \mathrm{H}^{*}\right]$} & \\
\hline & {$\left[\mathrm{L}+\mathrm{H}^{*}\right]$} & \\
\hline & {$\left[\mathrm{L}+; \mathrm{H}^{*}\right]$} & \\
\hline ! $\mathrm{H}^{*}$ & {$\left[\mathrm{~L}+! \mathrm{H}^{*}\right]$} & \\
\hline
\end{tabular}

\begin{tabular}{|c|c|c|}
\hline ESTRUCTURA PROFUNDA & ESTRUCTURA SLPERFICLAL & NVEL ACUSTICO \\
\hline Invariante fonológica & Variantes & \begin{tabular}{|l|l} 
Esquema del contorno tonal \\
\end{tabular} \\
\hline \multirow{2}{*}{ / $\mathrm{H} \% /$} & {$\left[\mathrm{H}^{\%}\right.$ ] } & тм \\
\hline & {$[\mathrm{MH} \%]$} & \\
\hline \multirow{3}{*}{ L $\%$ / } & [L\%] & TM \\
\hline & [ML\%] & \\
\hline & {$\left[\mathrm{H}_{\mathrm{L}} \%\right.$ ] } & \\
\hline
\end{tabular}

De las pautas que subyacen a la propuesta ilustrada en las tablas 1 y 2 destacamos tres: $1^{\text {a) }}$ para valorar la mayor o menor relevancia de las diferencias tonales se considera el umbral de 1,5 St ofrecido por Rietveld y Gussenhoven (1985) y ratificado para el español por Pamies Bertrán et al. (2002). Solo cuando se llega o se supera ese umbral se considera que la diferencia es significativa (no en sentido estadístico); $2^{a}$ ) Los datos del análisis permiten

\footnotetext{
${ }^{4}$ Fueron creadas en el año 2006 por el grupo AMPER-Astur de la Universidad de Oviedo (López-Bobo et al. 2007). Licencia de Matlab no 256105.
} 
aislar variantes e invariantes de los acentos tonales (tabla 1) y de los tonos de frontera (tabla 2); y $3^{\text {a) }}$ Para los acentos de frontera es fundamental considerar el tono medio (TM) puesto que serán altos $(\mathrm{H} \%)$ o bajos (L\%) dependiendo de si se supera o no el umbral de 1,5 St.

Aunque seguimos sosteniendo los principios de esta propuesta, el análisis posterior de una gran cantidad de datos y de más variedades de habla que las analizadas en ese momento, nos lleva a modificarla en el presente trabajo en los puntos siguientes. ${ }^{5}$

10) Las invariantes bitonales $/ \mathrm{L}^{*}+\mathrm{H} / \mathrm{y} / \mathrm{L}+\mathrm{H}^{*} /$ no solo aparecen en el primer acento como se proponía en Dorta (2013). Podemos encontrarlas también en el núcleo entonativo, solo que en este no siempre aparecen las mismas variantes como, por ejemplo, $\left[\mathrm{L}+>\mathrm{H}^{*}\right]$. La etiqueta $/ \mathrm{L}^{*}+\mathrm{H} /$ se usará siempre que la tónica quede baja o en ascenso, pero sin que le preceda una distancia significativa respecto del valle anterior y se dé un pico posterior. El acento bitonal $/ \mathrm{L}+\mathrm{H}^{*} /$, por su parte, se empleará siempre que se dé un ascenso significativo de la F0 (superior a 1,5 St) desde un valle precedente hasta la tónica. Con esta modificación, pues, las interrogativas con final circunflejo (alto-descendente) con tónica alta quedarán caracterizadas con el acento bitonal $/ \mathrm{L}+\mathrm{H}^{*} /$ (y no siempre $/ \mathrm{H}^{*} /$, como sostiene Dorta 2013) siempre que aparezcan precedidas de una subida significativa hasta la tónica; si esto no sucede quedarán caracterizadas por $/ \mathrm{H}^{*} /$; en ambos casos terminarán con $/ \mathrm{L} \% /$. No obstante, seguimos considerando, como en 2013 , que $/ \mathrm{H}^{*} /$ puede tener entre sus variantes $\left[\mathrm{L}+\mathrm{H}^{*}\right]$ y $\left[\mathrm{L}_{+} \mathrm{H}^{*}\right]$ puesto que en estos casos se implica que se da un valle anterior, pero la distancia hasta la tónica no alcanza el umbral por lo que no se contradice la definición de la invariante. Lo dicho valdría igualmente para los casos en que una interrogativa termina con ascenso, como sucede en diversas variedades, como el castellano o el mexicano, según se vio en la introducción.

$\left.2^{\circ}\right)$ En lo que respecta a los tonos de frontera, hemos comprobado que la distinción de solo dos tonos / $\mathrm{H} \% /$ y /L\%/ que se hacía en 2013 es insuficiente, sobre todo si queremos aplicarla al tono de frontera inicial y no solo final. Por ello, proponemos tres tonos, tanto para la frontera inicial como para la final y, asimismo, modificamos la relación y definición de las variantes. ${ }^{6}$

$/ \% \mathrm{H} /$ o / $\mathrm{H} \% /$. El inicio o final de la F0 se sitúa significativamente por encima del TM: $[\% \mathrm{H}]$ o $[\mathrm{H} \%]$.

/\%M/ o /M\%/. La F0 se sitúa en el TM o muy próximo a este sin que la diferencia alcance el umbral. Sus variantes son:

$[\% \mathrm{M}]$ o $[\mathrm{M} \%]$ : la F0 se inicia o termina en el TM sin movimiento anterior o posterior ascendente o descendente.

[\%MH\%] o [MH\%]: se da esta variante cuando la F0 tiene trayectoria ascendente

\footnotetext{
${ }^{5}$ Con todo, los cambios que proponemos siguen siendo provisionales, puesto que la propuesta se irá revisando continuamente a medida que avancemos en el análisis de las variedades del español que consideramos en el proyecto ya citado en el que se enmarca este trabajo.

${ }^{6}$ Dada la provisionalidad de la propuesta, no descartamos que aparezcan nuevas variantes.
} 
pero el inicio o final no llega a superar el umbral respecto del TM por lo que la F0 queda truncada en torno a este.

[\%ML] o [ML\%]: la trayectoria de la F0 es descendente pero el inicio o final no queda significativamente por debajo del TM.

3o) /\%L/ o /L\%/. El inicio de la F0 se sitúa significativamente por debajo del TM. Tiene dos variantes:

[\%L] o [L\%]: la F0 se sitúa significativamente por debajo del TM.

$\left[\mathrm{H}_{\mathrm{L}} \%\right]$ : como en la propuesta de Dorta (2013), se propone para los finales agudos de las interrogativas circunflejas donde la F0 queda truncada en una frecuencia alta pero subyace el patrón descendente de dichas interrogativas.

\section{Resultados}

\subsection{Declarativas}

\subsubsection{Datos generales}

La configuración tonal de las declarativas se caracteriza por un descenso al final más abrupto en voz femenina que en los hombres. El rango tonal medio (RTM) en que se mueven las curvas (tabla 3) es 4 St más amplio en las mujeres que en los hombres y el tono medio (TM) se sitúa 6,9 St por encima en voz femenina. Por otra parte, el mínimo de F0 se localiza 1,9 St y 5,3 St por debajo del TM, en hombres y mujeres, respectivamente y la diferencia entre los inicios y finales medios arroja pendientes descendentes con valores muy similares en los dos sexos y en los dos tipos de finales encontrados (entre $-4,4 \mathrm{y}-5 \mathrm{St}$ ).

Tabla 3. Valores medios generales de las declarativas

\begin{tabular}{|c|c|c|c|c|}
\hline & \multicolumn{4}{|c|}{ Datos generales de las declarativas } \\
\hline & \multicolumn{2}{|c|}{ Hombres } & \multicolumn{2}{|c|}{ Mujeres } \\
\hline Mínimo F0 & \multicolumn{2}{|c|}{$108 \mathrm{~Hz}$} & \multicolumn{2}{|c|}{$133 \mathrm{~Hz}$} \\
\hline Máximo F0 & \multicolumn{2}{|c|}{$140 \mathrm{~Hz}$} & \multicolumn{2}{|c|}{$218 \mathrm{~Hz}$} \\
\hline RTM & \multicolumn{2}{|c|}{$4,5 \mathrm{St}$} & \multicolumn{2}{|c|}{$8,5 \mathrm{St}$} \\
\hline TM & \multicolumn{2}{|c|}{$121 \mathrm{~Hz}$} & \multicolumn{2}{|c|}{$181 \mathrm{~Hz}$} \\
\hline \multirow[t]{2}{*}{ Pendiente I-F } & Final agudo (AT) & Final llano (ATA) & Final agudo (AT) & Final llano (ATA) \\
\hline & $-4,4 \mathrm{St}$ & $-4,9 \mathrm{St}$ & $-5 \mathrm{St}$ & $-4,5 \mathrm{St}$ \\
\hline
\end{tabular}




\subsubsection{Declarativas con final agudo}

Las declarativas con final agudo (tabla 4) se inician mayoritariamente con un tono de frontera alto $/ \% \mathrm{H} /$ o medio $/ \% \mathrm{M} /$ dependiendo de si el inicio se sitúa significativamente por encima del TM $[\% \mathrm{H}]$ o si se coloca alto, pero sin llegar a superar el TM [\%MH]; en las mujeres, además, aparece esporádicamente un tono bajo /L\%/. Separando los inicios TA y ATA se observa que en las mujeres es más frecuente la variante $[\% \mathrm{H}]$ cuando las frases comienzan por TA, mientras que $[\% \mathrm{MH}]$ predomina cuando comienzan por ATA. En los hombres, en cambio, es más frecuente $[\% \mathrm{H}]$ en ambos comienzos.

En las curvas estilizadas de la figura $3^{7}$ se puede observar que la F0 desciende desde un tono alto en el primer acento tonal, o asciende hasta un primer pico que se sincroniza con la tónica o con la postónica. En las mujeres (tabla 4) predomina el acento monotonal alto $/ \mathrm{H}^{*} /$ y en los hombres el bitonal $/ \mathrm{L}^{*}+\mathrm{H} /$; en este último caso, la F0 queda baja en la tónica, o bien se inicia el ascenso en la acentuada, pero la diferencia respecto del valle anterior no supera el umbral (Dorta 2013). Además de estos dos acentos mayoritarios, hombres y mujeres usan otros, como $/ \mathrm{L}_{+} \mathrm{H}^{*} /$ : los primeros con la variante $\left[\mathrm{L}+>\mathrm{H}^{*}\right]$ en la que la tónica es significativamente alta respecto del valle anterior, pero el ascenso de la F0 culmina después de la tónica (Dorta 2013); las mujeres emplean esta variante y más esporádicamente [L+H*] en donde el ascenso de la F0 culmina en la tónica donde se da el pico tonal (Dorta 2013).

Tabla 4. Etiquetado fonético-fonológico de las declarativas con final agudo

\begin{tabular}{|c|c|c|c|c|c|c|c|c|}
\hline & \multicolumn{8}{|c|}{ Declarativas con final agudo } \\
\hline & \multicolumn{2}{|c|}{ Tono de frontera inicial } & \multicolumn{2}{|c|}{ Acento inicial } & \multicolumn{2}{|c|}{ Acento nuclear } & \multicolumn{2}{|c|}{ Tono de frontera fina } \\
\hline & Inv. & Var. & Inv. & Var. & Inv. & Var. & Inv. & Var. \\
\hline \multirow{3}{*}{ 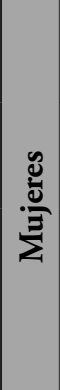 } & $\begin{array}{c}\% \mathbf{H} \\
(46,7 \%)\end{array}$ & $\begin{array}{c}\% \mathbf{H} \\
(46,7 \%)\end{array}$ & $\begin{array}{c}\mathbf{H}^{*} \\
(53,3 \%)\end{array}$ & $\begin{array}{c}\mathbf{H}^{*} \\
(53,3 \%)\end{array}$ & \multirow[t]{3}{*}{$\begin{array}{c}\mathbf{L}^{*} \\
(100 \%)\end{array}$} & \multirow[t]{3}{*}{$\begin{array}{c}\mathbf{L}^{*} \\
(100 \%)\end{array}$} & $\begin{array}{c}\mathbf{L} \% \\
(86,7 \%)\end{array}$ & $\begin{array}{c}\mathbf{L} \% \\
(86,7 \%)\end{array}$ \\
\hline & $\begin{array}{c}\% \mathrm{M} \\
(40 \%)\end{array}$ & $\begin{array}{l}\% \mathrm{MH} \\
(40 \%)\end{array}$ & $\begin{array}{c}\mathrm{L}^{*}+\mathrm{H} \\
(26,7 \%)\end{array}$ & $\begin{array}{c}\mathrm{L}^{*}+\mathrm{H} \\
(26,7 \%)\end{array}$ & & & \multirow[t]{2}{*}{$\begin{array}{c}\text { M\% } \\
(13,3 \%)\end{array}$} & \multirow[t]{2}{*}{$\begin{array}{c}\text { ML\% } \\
(13,3 \%)\end{array}$} \\
\hline & $\begin{array}{c}\% \mathrm{~L} \\
(11,4 \%)\end{array}$ & $\begin{array}{c}\% \mathrm{~L} \\
(13,3 \%)\end{array}$ & $\begin{array}{l}\mathrm{L}+\mathrm{H}^{*} \\
(20 \%)\end{array}$ & $\begin{array}{c}\mathrm{L}+>\mathrm{H}^{*} \\
(13,3 \%) \\
\mathrm{L}+\mathrm{H}^{*} \\
(6,7 \%)\end{array}$ & & & & \\
\hline
\end{tabular}

\footnotetext{
${ }^{7}$ La figura 3 es una representación estilizada de la media de oraciones con final agudo que comienzan por TA y por ATA: $\mathrm{I}$ = inicio; $\mathrm{P} 1=$ pico 1; PreN= pretónica nuclear; TN tónica nuclear; $\mathrm{F}=$ final. En figuras posteriores se usará, además, Pos $\mathrm{N}=$ postónica nuclear.
} 


\begin{tabular}{|c|c|c|c|c|c|c|c|c|}
\hline \multirow{3}{*}{ 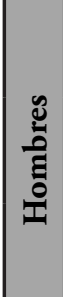 } & $\begin{array}{c}\text { \%H } \\
(85,7 \%)\end{array}$ & $\begin{array}{c}\mathbf{\% H} \\
(85,7 \%)\end{array}$ & $\begin{array}{c}\mathbf{L}^{*}+\mathbf{H} \\
(42,8 \%)\end{array}$ & $\begin{array}{c}\mathbf{L}^{*}+\mathbf{H} \\
(42,8 \%)\end{array}$ & \multirow[t]{3}{*}{$\begin{array}{c}\mathbf{L}^{*} \\
(100 \%)\end{array}$} & \multirow[t]{3}{*}{$\begin{array}{c}\mathbf{L}^{*} \\
(100 \%)\end{array}$} & $\begin{array}{c}\mathbf{L} \% \\
(71,4 \%)\end{array}$ & $\begin{array}{c}\mathbf{L} \% \\
(71,4 \%)\end{array}$ \\
\hline & \multirow[t]{2}{*}{$\begin{array}{c}\% \mathrm{M} \\
(14,3)\end{array}$} & \multirow[t]{2}{*}{$\begin{array}{c}\% \mathrm{MH} \\
(14,3 \%)\end{array}$} & $\begin{array}{c}\mathrm{H}^{*} \\
(28,6 \%)\end{array}$ & $\begin{array}{c}H^{*} \\
(28,6 \%)\end{array}$ & & & \multirow{2}{*}{$\begin{array}{c}\mathrm{M \%} \\
(28,6 \%)\end{array}$} & \multirow{2}{*}{$\begin{array}{c}\text { ML\% } \\
(28,6 \%)\end{array}$} \\
\hline & & & $\begin{array}{c}\mathrm{L}+\mathrm{H}^{*} \\
(28,6 \%)\end{array}$ & $\begin{array}{c}\mathrm{L}+>\mathrm{H}^{*} \\
(28,6 \%)\end{array}$ & & & & \\
\hline
\end{tabular}

Figura 3. Representación estilizada de declarativas con final agudo

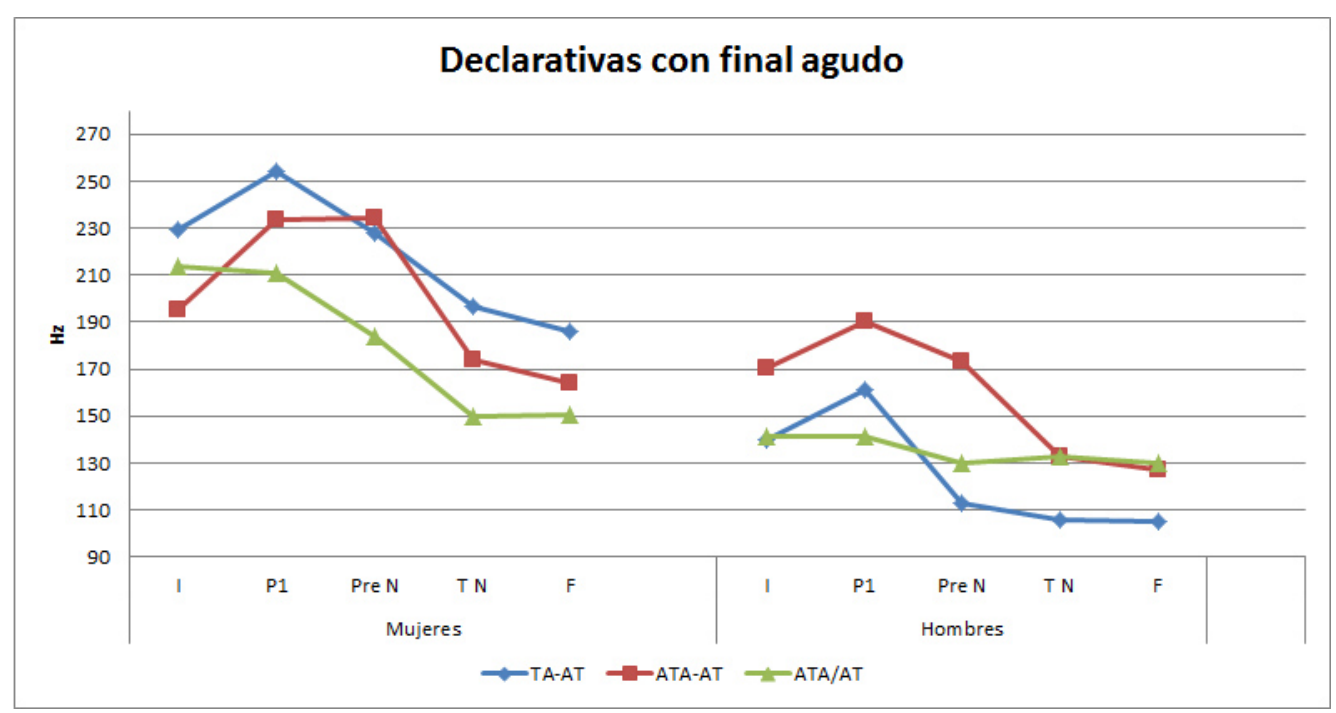

A partir del tono alto o del pico inicial, la F0 desciende hasta el final. La característica más destacada del descenso es que el salto desde la pretónica hasta la tónica nuclear es muy significativo en las mujeres, mientras que en los hombres no lo es, salvo excepciones; ${ }^{8}$ en cualquiera de los casos, la tónica queda baja /L*/ en el 100\% de los casos y desde ella continúa el descenso hasta el final absoluto, por lo que el tono de frontera final es mayoritariamente /L\%/ con la variante [L\%] en los dos sexos o, más esporádicamente, $/ \mathrm{M} \% /$ con la variante [ML\%]; en esta última, si bien el final es descendente, el descenso no llega a 1,5 St respecto del TM.

\footnotetext{
${ }^{8}$ Como sucede en las frases ATA-AT 2a en que el salto es de $-2,4 \mathrm{St}$.
} 


\subsubsection{Declarativas con final llano}

Las declarativas con final llano comienzan por un tono de frontera inicial alto $1 \% \mathrm{H} /$, medio $/ \% \mathrm{M} /$ o bajo $/ \% \mathrm{~L} /$ con las variantes $[\% \mathrm{H}],[\% \mathrm{MH}]$ y $[\% \mathrm{~L}]$, respectivamente. En los porcentajes generales de la tabla 5 se puede observar que la primera variante es la más frecuente en las mujeres, mientras que en los hombres los tonos alto y medio, con las referidas variantes, comparten el mismo porcentaje. Al separar los dos tipos de inicio, TA y ATA, observamos que en TA las mujeres usan preferentemente la variante $[\% \mathrm{H}]$ y los hombres $[\% \mathrm{M}]$; cuando las frases comienzan por ATA, las mujeres siguen empleando $[\% \mathrm{H}]$ $\mathrm{y}$, en cambio, los hombres [\%L]. Además de los tonos de frontera alto y medio, en los dos sexos encontramos un tono bajo /\%L/; al separar las oraciones según el tipo de inicio se observa que este tono no aparece en los hombres cuando las oraciones comienzan por TA y, en cambio, su frecuencia llega a superar a $[\% \mathrm{MH}]$ en las oraciones que comienzan por ATA.

En el primer acento, la trayectoria de la F0 (tabla 5 y figura 4) no coincide del todo con la que vimos en las oraciones con final agudo, pues si bien volvemos a encontrar en los dos sexos los tonos $/ \mathrm{H}^{*} /, / \mathrm{L}^{*}+\mathrm{H} / \mathrm{y} / \mathrm{L}+\mathrm{H}^{*} /$, su uso no es el mismo que en los agudos; además, solo los hombres, y de manera esporádica, usan /\%L/. En efecto, como se ve en los porcentajes de las tablas 4 y 5 , si en los agudos de las mujeres predomina el primero de estos acentos, en los llanos es mayoritario el segundo -tónica baja respecto del pico- y es muy esporádico el tercero con la variante $\left[\mathrm{L}+\mathrm{H}^{*}\right]-$ el pico se sincroniza con la tónica-. Por otra parte, en los hombres el acento tonal inicial más frecuente en los agudos es $/ \mathrm{L}^{*}+\mathrm{H} /$, en cambio en los llanos es $/ \mathrm{L}+\mathrm{H}^{*} /$.

Tabla 5. Etiquetado fonético-fonológico de las declarativas con final llano

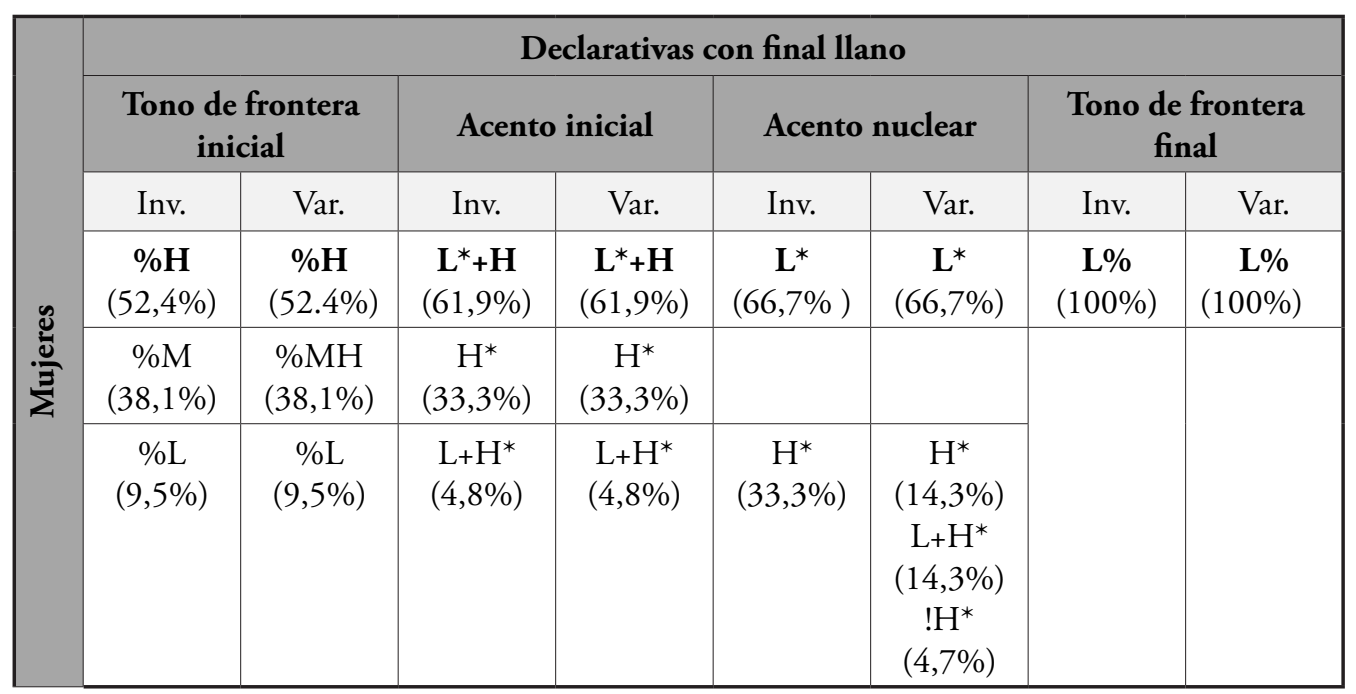




\begin{tabular}{|c|c|c|c|c|c|c|c|c|}
\hline \multirow{4}{*}{ 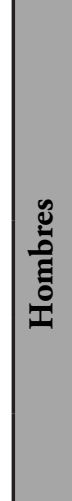 } & $\begin{array}{c}\mathbf{\%} \mathbf{H} \\
(37,2 \%)\end{array}$ & $\begin{array}{c}\mathbf{\% H} \\
(37,2 \%)\end{array}$ & $\begin{array}{l}\mathbf{L}+\mathbf{H}^{*} \\
(41 \%)\end{array}$ & $\begin{array}{c}\mathbf{L}+>\mathbf{H}^{*} \\
(29,6 \%) \\
\\
\mathrm{L}+\mathrm{H}^{*} \\
(11,4 \%)\end{array}$ & $\begin{array}{c}\mathbf{L}^{*} \\
(95,4 \%)\end{array}$ & $\begin{array}{c}\mathbf{L}^{*} \\
(95,4 \%)\end{array}$ & $\begin{array}{c}\mathbf{L} \% \\
(100 \%)\end{array}$ & $\begin{array}{c}\mathbf{L} \% \\
(100 \%)\end{array}$ \\
\hline & $\begin{array}{c}\mathbf{\% M} \\
(37,2 \%)\end{array}$ & $\begin{array}{c}\text { \%MH } \\
(37,2 \%)\end{array}$ & $\begin{array}{c}\mathrm{L}^{*}+\mathrm{H} \\
(20,4 \%)\end{array}$ & $\begin{array}{c}\mathrm{L}^{*}+\mathrm{H} \\
(20,4 \%)\end{array}$ & & & & \\
\hline & \multirow[t]{2}{*}{$\begin{array}{c}\% \mathrm{~L} \\
(25,6 \%)\end{array}$} & \multirow[t]{2}{*}{$\begin{array}{c}\% \mathrm{~L} \\
(25,6 \%)\end{array}$} & $\begin{array}{c}H^{*} \\
(31,8 \%)\end{array}$ & $\begin{array}{c}\mathrm{H}^{*} \\
(31,8 \%)\end{array}$ & $\begin{array}{c}H^{*} \\
(6,9 \%)\end{array}$ & $\begin{array}{l}\mathrm{L}+! \mathrm{H}^{*} \\
(2,3 \%)\end{array}$ & & \\
\hline & & & $\begin{array}{c}\mathrm{L}^{*} \\
(6,8 \%)\end{array}$ & $\begin{array}{c}L^{*} \\
(6,8 \%)\end{array}$ & $\begin{array}{c}\mathrm{L}^{*}+\mathrm{H} \\
(2,3 \%)\end{array}$ & $\begin{array}{c}\mathrm{L}^{*}+\mathrm{H} \\
(2,3 \%)\end{array}$ & & \\
\hline
\end{tabular}

Figura 4. Representación estilizada de las declarativas con final llano

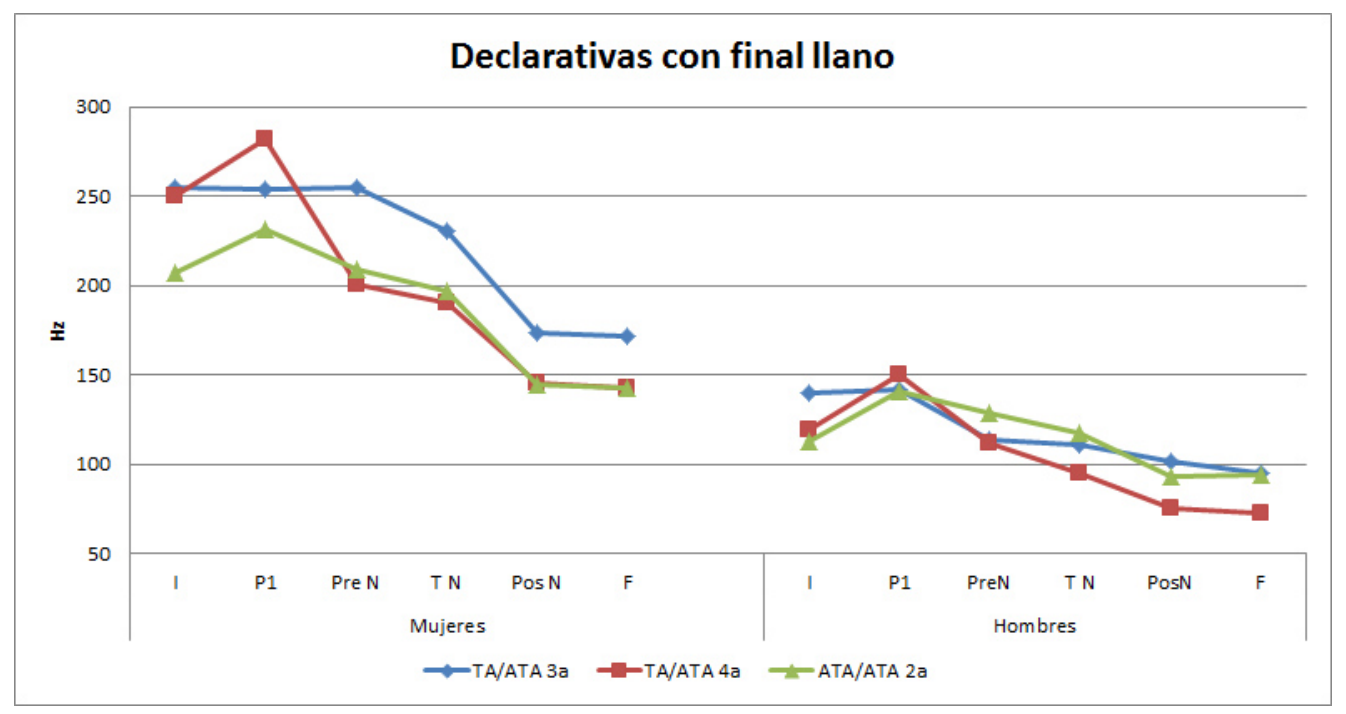

A partir del primer acento tonal, tanto en hombres como en mujeres, lo más frecuente es que la F0 descienda de manera progresiva o con picos poco significativos hasta el final, tal como se refleja en la ilustración que precede. No obstante, lo más relevante es que si bien la tónica nuclear se caracteriza por un tono bajo $/ \mathrm{L}^{*} /$, salvo excepciones, como veremos a continuación, la F0 experimenta siempre un salto muy abrupto (más en las mujeres que en los hombres) desde la tónica a la postónica y, de manera menos significativa, desde esta hasta el final terminando con un tono de frontera bajo /L\%/. Teniendo en cuenta las medias generales, la tónica nuclear de las frases con final llano se sitúa en frecuencias 
significativamente inferiores a la de los finales agudos $(2,4$ y $5 \mathrm{St}$, respectivamente en hombres y mujeres). Excepcionalmente, sobre todo en los hombres, se encontraron declarativas con final circunflejo en las que se da un pico que se alinea con la tónica nuclear a partir de la cual desciende la F0 hasta el final. En estos casos, por tanto, el tono nuclear y el acento de frontera final es $/ \mathrm{H}^{*} \mathrm{~L} \% /$ con las variantes y porcentajes que pueden verse en la tabla 5 .

\subsection{Interrogativas}

\subsubsection{Datos generales}

Una de las características más constantes de las interrogativas analizadas en este trabajo es que siempre terminan con ascenso de la F0 (figura 2), a diferencia de otro tipo de interrogativas, como las de Canarias o de Cuba, que terminan con final circunflejo o alto descendente (Dorta 2013). El comportamiento tonal más frecuente es el siguiente: en el núcleo entonativo de los finales agudos la tónica queda alta respecto del valle anterior y, a partir de ella, la F0 sube muy poco puesto que no hay sílabas posteriores que le permitan progresar. En los finales llanos, en cambio, la tónica queda en el valle o muy próxima a este y el ascenso de la F0 a partir de ella es más amplio que en los agudos puesto que progresa en la postónica y de esta hasta el final absoluto. En la tabla 6 incluimos algunos datos obtenidos a partir de las medias.

Tabla 6. Valores medios de las interrogativas

\begin{tabular}{|c|c|c|c|c|}
\hline & \multicolumn{4}{|c|}{ Datos generales de las interrogativas } \\
\hline & \multicolumn{2}{|c|}{ Hombres } & \multicolumn{2}{|c|}{ Mujeres } \\
\hline Mínimo F0 & \multicolumn{2}{|c|}{$119 \mathrm{~Hz}$} & \multicolumn{2}{|c|}{$154 \mathrm{~Hz}$} \\
\hline Máximo F0 & \multicolumn{2}{|c|}{$155 \mathrm{~Hz}$} & \multicolumn{2}{|c|}{$229 \mathrm{~Hz}$} \\
\hline RTM & \multicolumn{2}{|c|}{$4,6 \mathrm{St}$} & \multicolumn{2}{|c|}{$6,8 \mathrm{St}$} \\
\hline $\mathrm{TM}$ & \multicolumn{2}{|c|}{$133 \mathrm{~Hz}$} & \multicolumn{2}{|c|}{$197 \mathrm{~Hz}$} \\
\hline \multirow[t]{2}{*}{ Pendiente I-F en St } & $\begin{array}{c}\text { Final agudo } \\
\text { (AT) }\end{array}$ & $\begin{array}{l}\text { Final llano } \\
\text { (ATA) }\end{array}$ & $\begin{array}{l}\text { Final agudo } \\
\text { (AT) }\end{array}$ & $\begin{array}{c}\text { Final llano } \\
\text { (ATA) }\end{array}$ \\
\hline & 5,1 & 7,1 & 2,8 & 3,6 \\
\hline
\end{tabular}

De los datos de la tabla se desprende que, como en las declarativas, el RTM de las mujeres es más amplio que el de los hombres (2,2 St de diferencia), que el TM de aquellas se sitúa significativamente por encima de la voz masculina $(6,8 \mathrm{St})$ y que el mínimo de F0 
se localiza por debajo del TM en frecuencias similares a dicha modalidad, esto es, 1,9 St y 4,3 St en hombres y mujeres, respectivamente. En cambio, el TM se sitúa 1,6 y 1,5 St por encima de las declarativas en hombres y mujeres, respectivamente y la pendiente I-F generada en esta modalidad es ascendente (no descendente) y más inclinada en los finales llanos que en los agudos.

\subsubsection{Interrogativas con final agudo}

Todas las interrogativas con final agudo analizadas comienzan por ATA en las mujeres y por ATA/AT y TA en los hombres. En general, como puede verse en la tabla 7 , se inician mayoritariamente con un tono de frontera medio $/ \% \mathrm{M} /$ en las primeras, con la variante $[\% \mathrm{MH}]$, y con un tono bajo $/ \% \mathrm{~L} / \mathrm{en}$ los segundos. Con todo, el porcentaje del tono medio en los hombres (41,7\%) se aproxima bastante al mayoritario /\%L/ (58,3\%). Al separar en estos últimos las oraciones por el tipo de inicio TA o ATA/AT, se observó que en ambos casos aparecen los dos tonos citados.

En el primer acento el comportamiento más frecuente es el ascenso de la F0 hasta un pico tonal (figura 5). Ahora bien, en las mujeres predomina $/ \mathrm{L}^{*}+\mathrm{H} /$ con la variante $\left[\mathrm{L}^{*}+\mathrm{H}\right]$-tónica baja y pico posterior-, mientras que en los hombres este acento es esporádico. En estos últimos es más frecuente $/ \mathrm{L}+\mathrm{H}^{*} /$ con las variantes $\left[\mathrm{L}+\mathrm{H}^{*}\right]-\mathrm{el}$ pico se alinea con la tónica- ${ }^{-}\left[\mathrm{L}+>\mathrm{H}^{*}\right]-$ el pico se pospone- en porcentajes muy próximos (tabla 7); hay que destacar que / $\mathrm{L}+\mathrm{H}^{*} /$ también está presente en las interrogativas de las mujeres con la variante predominante $\left[\mathrm{L}+>\mathrm{H}^{*}\right]$. En ambos sexos se utiliza más esporádicamente el tono bajo $/ \mathrm{L}^{*} /$. En comparación con el primer acento tonal de las declarativas con final agudo (tabla 4), los hechos más destacados son: no hemos encontrado el tono $/ \mathrm{H}^{*} /$ inicial que aparecía de manera mayoritaria en las mujeres y en menor porcentaje en los hombres $\mathrm{y}$, por otra parte, mientras que en las declarativas de los hombres el primer acento era mayoritariamente $/ \mathrm{L}^{*}+\mathrm{H} /(42,8 \%)$, en las interrogativas es $/ \mathrm{L}+\mathrm{H}^{*} /(70,8 \%)$, que también aparecía en las declarativas con mucha menos frecuencia $(28,6 \%)$. 
Tabla 7. Etiquetado fonético-fonológico de las interrogativas con final agudo

\begin{tabular}{|c|c|c|c|c|c|c|c|c|}
\hline \multicolumn{8}{|c|}{ Interrogativas con final agudo en corpus situacional } & \multirow[b]{3}{*}{ Var. } \\
\hline \multicolumn{2}{|c|}{$\%$ Tono inicial } & \multicolumn{2}{|c|}{ Acento inicial } & \multicolumn{2}{|c|}{ Acento nuclear } & \multicolumn{2}{|c|}{ Tono final \% } & \\
\hline Informantes & Inv. & Var. & Inv. & Var. & Inv. & Var. & Inv. & \\
\hline \multirow{4}{*}{ 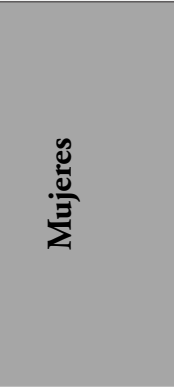 } & $\begin{array}{c}\text { \%M } \\
(72,7 \%)\end{array}$ & $\begin{array}{c}\text { \%MH } \\
(72,7 \%)\end{array}$ & $\begin{array}{c}\mathbf{L}^{*}+\mathbf{H} \\
(45,4 \%)\end{array}$ & $\begin{array}{c}\mathbf{L}^{*}+\mathbf{H} \\
(45,4 \%)\end{array}$ & $\begin{array}{c}\mathbf{L}+\mathbf{H}^{*} \\
(81,8 \%)\end{array}$ & $\begin{array}{c}\mathbf{L}+\mathbf{H}^{*} \\
(81,8 \%)\end{array}$ & \multirow[t]{4}{*}{$\begin{array}{c}\mathbf{H} \% \\
(100 \%)\end{array}$} & \multirow[t]{4}{*}{$\begin{array}{c}\mathbf{H} \% \\
(100 \%)\end{array}$} \\
\hline & \multirow[t]{3}{*}{$\begin{array}{c}\% \mathrm{H} \\
(27,3 \%)\end{array}$} & \multirow[t]{3}{*}{$\begin{array}{c}\% \mathrm{H} \\
(27,3 \%)\end{array}$} & $\begin{array}{c}\mathrm{L}+\mathrm{H}^{*} \\
(36,4 \%)\end{array}$ & $\begin{array}{c}\mathrm{L}+>\mathrm{H}^{*} \\
(27,3 \%)\end{array}$ & \multirow[t]{3}{*}{$\begin{array}{c}\mathrm{L}^{*} \\
(18,2 \%)\end{array}$} & \multirow[t]{3}{*}{$\begin{array}{c}\mathrm{L}^{*} \\
(18,2 \%)\end{array}$} & & \\
\hline & & & & $\begin{array}{c}\mathrm{L}+\mathrm{H}^{*} \\
(9,1 \%)\end{array}$ & & & & \\
\hline & & & $\begin{array}{c}L^{*} \\
(18,2 \%)\end{array}$ & $\begin{array}{c}\mathrm{L}^{*} \\
(18,2 \%)\end{array}$ & & & & \\
\hline \multirow{4}{*}{ 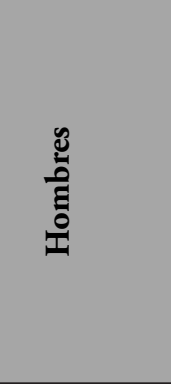 } & \multirow[t]{3}{*}{$\begin{array}{c}\text { \%L } \\
(58,3 \%)\end{array}$} & \multirow[t]{3}{*}{$\begin{array}{c}\text { \%L } \\
(58,3 \%)\end{array}$} & $\begin{array}{c}\mathbf{L}+\mathbf{H}^{*} \\
(70,8 \%)\end{array}$ & $\begin{array}{c}\mathbf{L}+\mathbf{H}^{*} \\
(37,5 \%)\end{array}$ & \multirow[t]{2}{*}{$\begin{array}{c}\mathbf{H}^{*} \\
(54,2)\end{array}$} & $\begin{array}{c}\mathbf{H}^{*} \\
(37,5 \%)\end{array}$ & \multirow[t]{2}{*}{$\begin{array}{c}\mathbf{H \%} \\
(95,8 \%)\end{array}$} & \multirow[t]{2}{*}{$\begin{array}{c}\mathbf{H} \% \\
(95,8 \%)\end{array}$} \\
\hline & & & & $\begin{array}{c}\mathrm{L}+>\mathrm{H}^{*} \\
(33,3 \%)\end{array}$ & & $\begin{array}{c}\mathfrak{j}^{\mathbf{H}^{*}} \\
(16,7 \%)\end{array}$ & & \\
\hline & & & $\begin{array}{c}L^{*} \\
(16,7 \%)\end{array}$ & $\begin{array}{c}L^{*} \\
(16,7 \%)\end{array}$ & \multirow[t]{2}{*}{$\begin{array}{c}\mathrm{L}+\mathrm{H}^{*} \\
(45,8 \%)\end{array}$} & \multirow[t]{2}{*}{$\begin{array}{c}\mathrm{L}+\mathrm{H}^{*} \\
(45,8 \%)\end{array}$} & \multirow[t]{2}{*}{$\begin{array}{c}\mathrm{M} \% \\
(4,2 \%)\end{array}$} & \multirow[t]{2}{*}{$\begin{array}{l}\text { MH\% } \\
(4,2 \%)\end{array}$} \\
\hline & $\begin{array}{c}\% \mathrm{M} \\
(41,7 \%)\end{array}$ & $\begin{array}{c}\% \mathrm{M} \\
(41,7 \%)\end{array}$ & $\begin{array}{c}\mathrm{L}^{*}+\mathrm{H} \\
(12,5 \%)\end{array}$ & $\begin{array}{c}\mathrm{L}^{*}+\mathrm{H} \\
(12,5 \%)\end{array}$ & & & & \\
\hline
\end{tabular}

Figura 5. Representación estilizada de las interrogativas con final agudo

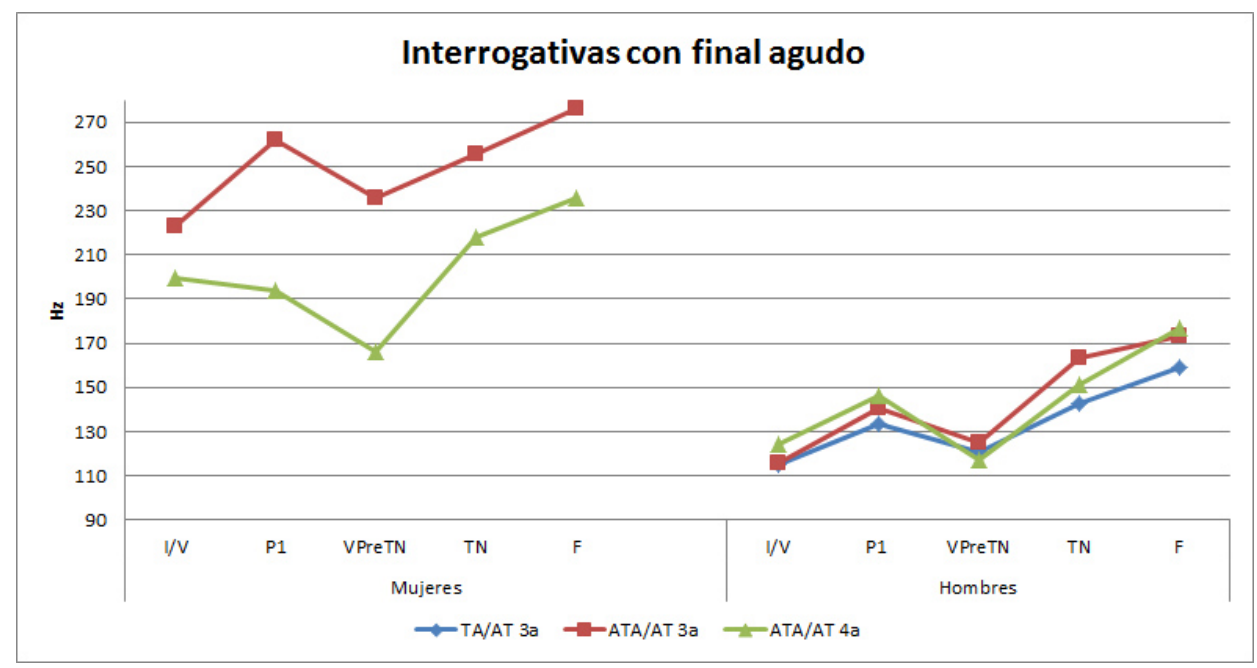


Desde el primer pico o, menos frecuentemente, desde la tónica del primer acento, el comportamiento de la F0, salvo excepciones, es el siguiente: descenso sin picos significativos hasta un valle situado en la pretónica nuclear. ${ }^{9}$ A partir del valle, en las mujeres experimenta, salvo excepciones, un ascenso relevante hasta la tónica nuclear que continúa hasta el final por lo que esta, a diferencia de las declarativas, tiene un tono alto $/ \mathrm{L}+\mathrm{H}^{*} /$-salto significativo del valle precedente a la tónica-, sea cual sea su inicio, y un tono de frontera $/ \mathrm{H} \% / .^{10} \mathrm{En}$ los hombres, por su parte, la tónica nuclear también queda alta pero, a diferencia de las mujeres, utilizan el tono $/ \mathrm{H}^{*} /$ y el bitonal $/ \mathrm{L}+\mathrm{H}^{*} /$ debido a que en el primer caso no se da un salto significativo desde el valle a la tónica, mientras que en el segundo sí. Cuando emplean $/ \mathrm{H}^{*} /$ lo realizan como $\left[\mathrm{H}^{*}\right]$ y en menor medida como $\left[{ }_{i} \mathrm{H}^{*}\right]$; la diferencia entre ambas variantes estriba en que en la segunda se da un escalonamiento ascendente. En cuanto al tono de frontera final utilizan, como las mujeres, / $\mathrm{H} \% /$ y muy esporádicamente $/ \mathrm{M} \% /$ con la variante $[\mathrm{MH} \%]$ pues, aunque en este último caso el final es ascendente, no llega a superar significativamente el TM. La frecuencia media del valle tonal a partir del cual se inicia el ascenso nuclear se sitúa en los hombres en $121 \mathrm{~Hz}$, es decir, prácticamente en el mínimo de F0 (solo 0,3 St por encima); en las mujeres, en cambio, se coloca en $198 \mathrm{~Hz}$ (4,3 St por encima del mínimo). El salto medio desde el valle a la tónica es de 3,2 y 2,7 St en hombres y mujeres, respectivamente y el que se da desde el valle hasta el final es de 5,1 y 4,3 St, respectivamente. En definitiva, lo más frecuente en el final de las interrogativas con final agudo es un ascenso significativo hasta la tónica y de aquí hasta el final.

\subsubsection{Interrogativas con final llano}

En este caso, mujeres y hombres inician mayoritariamente sus enunciados con un tono de frontera alto $/ \% \mathrm{H} /$ y medio $/ \% \mathrm{M} /$, respectivamente (tabla 8 ). Hay que destacar que en las mujeres $[\% \mathrm{H}]$ es la variante más frecuente en los inicios TA y ATA; en cambio, en los hombres la variante $[\% \mathrm{M}]$ solo es mayoritaria en los inicios TA, pero no en ATA donde predomina $[\% \mathrm{~L}]$. El porcentaje de aparición del tono de frontera bajo $/ \% \mathrm{~L} /$, en cambio, es muy bajo en las mujeres y relativamente alto en los hombres; en las primeras solo se realiza en las oraciones que se inician por TA, mientras que en los hombres se da en todos los inicios, pero más en las frases que comienzan por ATA.

En el acento inicial los hombres vuelven a utilizar más frecuentemente $/ \mathrm{L}+\mathrm{H}^{*} /$, como en las interrogativas con final agudo -la F0 sube desde el inicio o desde un valle anterior hasta la tónica donde se da el pico tonal-, si bien en un porcentaje menor. Usan, además, los tonos $/ \mathrm{L}^{*}+\mathrm{H} /, / \mathrm{H}^{*} /$ y $/ \mathrm{L}^{*} /$ en los porcentajes que se muestran en la tabla 8 . Las mujeres, en cambio, emplean preferentemente el tono $/ \mathrm{H}^{*} /$ (no utilizado en las interrogativas con final agudo), aunque solo en la mitad de los casos; en menor medida usan $/ \mathrm{L}^{*} / \mathrm{y} / \mathrm{L}^{*}+\mathrm{H} /$.

\footnotetext{
${ }^{9}$ Excepcionalmente, el valle se da en la tónica del segundo acento.

${ }^{10}$ Como se ve en la tabla 7, excepcionalmente la tónica queda baja en las mujeres (18,2\%), pero después la F0 asciende por lo que el final es ascendente $/ \mathrm{H} \% /$ como en el resto de las oraciones.
} 
Tabla 8. Etiquetado fonético-fonológico de las interrogativas con final llano

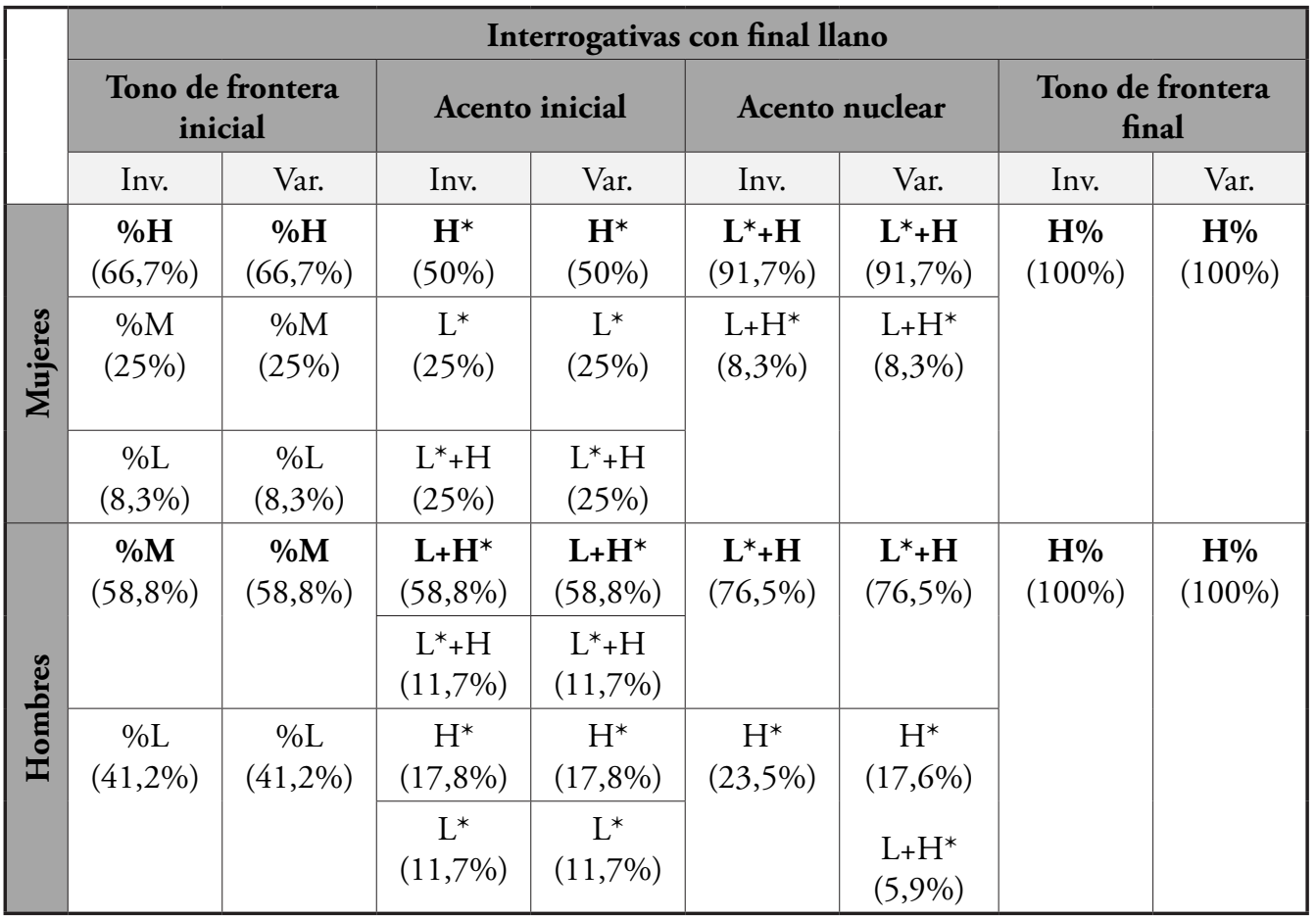

Figura 6. Representación estilizada de las interrogativas con final llano

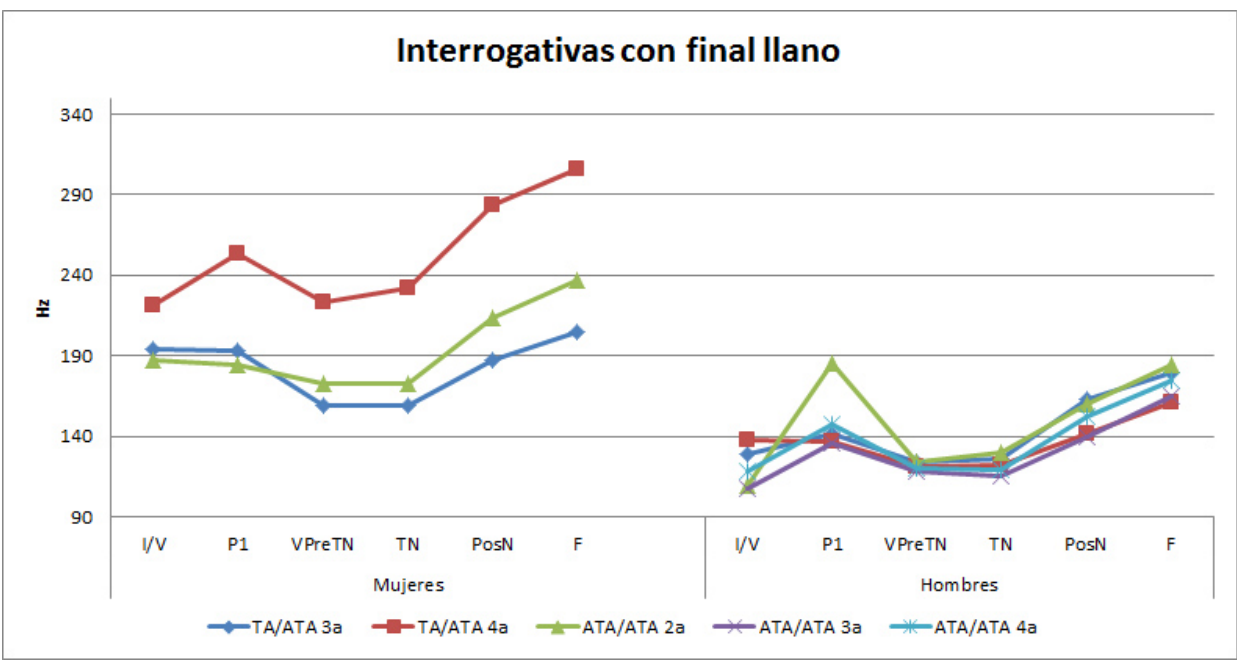


A partir del primer acento tonal, el comportamiento tonal más frecuente de las interrogativas con final llano (figura 6) es el descenso de la F0 desde la tónica inicial o desde el primer pico hasta la pretónica nuclear o más esporádicamente hasta la tónica-valle tonal- ${ }^{11}$ En este caso, entre tónica y pretónica nuclear no se dan diferencias significativas por encima del umbral y, por tanto, ambas quedan bajas ${ }^{12}$-la tónica nuclear queda $0,3 \mathrm{y}$ 2,5 St por encima del mínimo de F0 en hombres y mujeres, respectivamente- ${ }^{-}$Finalmente, desde el valle tonal, la F0 sube bruscamente hasta la postónica (una media de 3,8 y 4,4 St en hombres y mujeres, respectivamente) y desde ahí hasta el final (una media de 1,9 y 1,8 St en ambos sexos, respectivamente).

Por tanto, como puede verse en la tabla 8, las oraciones con final llano se caracterizan, salvo excepciones, por un acento tonal nuclear bitonal $/ \mathrm{L}^{*}+\mathrm{H} /$ con la realización $\left[\mathrm{L}^{*}+\mathrm{H}\right]$ puesto que la tónica queda baja y se da una subida posterior significativa hasta el final por lo que el acento de frontera final es siempre / $\mathrm{H} \% /$ con la única variante [H\%]. En definitiva, pues, aunque las oraciones con final llano terminan con ascenso final igual que las que tienen final agudo, la diferencia fundamental entre ambas es que en los agudos la tónica, salvo excepciones, queda alta y en los llanos, salvo excepciones, baja.

\section{Discusión De los RESUltados}

La comparación de los resultados obtenidos con los datos ofrecidos por otros autores del español mexicano permite ver que en las declarativas el pico inicial, cuando se da propiamente, no siempre se pospone a la primera sílaba acentuada (Sosa 1999), ni tampoco se alinea siempre con la tónica en los sintagmas con final agudo y se pospone en los finales llanos (Congosto 2012). Si tenemos en cuenta todos los casos en que la tónica queda alta, con independencia de si precede o no un valle anterior significativo, observamos que en las mujeres tal hecho sucede en un $73,3 \%$ y en un $38,1 \%$ de las oraciones con final agudo y llano, respectivamente; en el resto de los casos queda baja. Por otra parte, el pico tonal inicial, cuando se da, se pospone solo en un 13,3\% de los casos (véase la tabla 4). En los hombres, la tónica queda alta en un $57,2 \%$ y un $72,8 \%$ de las oraciones con final agudo y llano, respectivamente; de estos casos, solo en un $28,6 \%$ y $29,6 \%$ se pospone el pico en agudos y llanos, respectivamente (tablas 4 y 5). Por otra parte, la F0 en esta modalidad, salvo excepciones, experimenta un descenso hasta el final, como en las declarativas mexicanas (Sosa 1999; Quilis 1985; Mota et al. 2010; Congosto 2012) y en el espańol general, por lo

\footnotetext{
${ }^{11}$ En las mujeres se registró esporádicamente un segundo pico prenuclear cuya subida desde el valle anterior supera el umbral $(1,6 \mathrm{St})$; es a partir de este pico desde donde se produce el descenso hasta la pretónica nuclear. En otros casos, tanto en mujeres como en hombres, se da un segundo pico pero sin que la subida desde el valle anterior supere 1 St.

${ }^{12}$ En la figura 6, aunque la TN de las frases TA/ATA4a parece muy alta respecto del valle precedente, realmente sube solo 1,2 St por lo que no llega al umbral diferencial. Como puede verse en la tabla 8 , solo en un $8,3 \%$ y en un $23,5 \%$ de las interrogativas con final llano de las mujeres y hombres, respectivamente, la tónica queda alta.
} 
que el tono nuclear y el tono de frontera se etiquetan, salvo excepciones, como /L* L\%/. Se trata de un descenso sin picos significativos y, según las medias generales, tanto la tónica nuclear como el final absoluto de las frases con final agudo (AT) se sitúan en frecuencias significativamente superiores a las de los finales llanos. Es destacable en los finales agudos de las mujeres el salto significativo de la pretónica a la tónica y en los finales llanos, sobre todo de las mujeres, el salto desde la tónica a la postónica. Por último, excepcionalmente hemos encontrado, solo en los finales llanos de mujeres y hombres, un movimiento circunflejo $/ \mathrm{H}^{*}$ L\%/ en el núcleo tonemático de esta modalidad que, o bien no se menciona en habla formal de informantes mexicanos (Congosto 2012) o es registrado por otros autores en el habla neutra de México (Sosa 1999; Mota et al. 2010), en el habla popular (Matluck 1965), en hombres y mujeres del nivel bajo (Martín Butragueño 2004) o en estudiantes universitarios (Quilis 1985).

En lo que respecta a las interrogativas, nuestros resultados ratifican que el TM en esta modalidad es más elevado que el de las declarativas, pero el tono de frontera inicial no es siempre /\%H/ (Sosa 1999). Teniendo en cuenta el TM en esta modalidad, ese tono de frontera alto $/ \% \mathrm{H} /$ se da solo en las mujeres en un $27,3 \%$ y $66,7 \%$ de las interrogativas con final agudo y llano, respectivamente (tablas 7 y 8). En cuanto al pico inicial, nuestros resultados evidencian que, cuando se da propiamente, no siempre se pospone a la tónica (Sosa 1999; Congosto 2012), pues esto sucede solo en un $27,3 \%$ y en un $33,3 \%$ de las interrogativas con final agudo de mujeres y hombres, respectivamente (tabla 7). En lo que respecta al núcleo entonativo, nuestros resultados indican que la tónica no siempre queda baja próxima a la línea de base tonal y a partir de ella se da un ascenso muy amplio $\left(\mathrm{L}^{*}+\mathrm{H}\right.$ $\mathrm{H} \%$ ), como indica Sosa (1999), o que la tónica alcanza un mínimo tonal que continúa en la postónica para elevarse luego bruscamente $\left(\mathrm{L}^{*} \mathrm{LH} \%\right)$, como señalan otros autores (Mota et al. 2010). Salvo raras excepciones en los finales agudos, las interrogativas de San Antonio terminan en un tono de frontera alto $/ \mathrm{H} \% /$ pero, teniendo en cuenta la separación de las oraciones por su final, agudo o llano, se observó lo siguiente:

10) En los finales agudos (tabla 7) las mujeres emplean, salvo excepciones, el acento tonal nuclear $/ \mathrm{L}_{+} \mathrm{H}^{*} /$ con la realización más frecuente $\left[\mathrm{L}+\mathrm{H}^{*}\right]$, es decir, la tónica queda alta tras un ascenso significativo desde un valle tonal precedente, por lo que el acento nuclear y el tono de frontera final son $/ \mathrm{L}+\mathrm{H}^{*} \mathrm{H} \% /$. En estos mismos finales, los hombres emplean el acento monotonal seguido de un tono de frontera alto $/ \mathrm{H}^{*} \mathrm{H} \% /$ y el bitonal $/ \mathrm{L}+\mathrm{H}^{*} \mathrm{H} \% /$ en porcentajes muy próximos.

$2^{\circ}$ ) En cambio, en las oraciones con final llano (tabla 8), predomina, tanto en los hombres como en las mujeres, el acento $/ \mathrm{L}^{*}+\mathrm{H} /$ con tónica baja y subida posterior, por lo que en estos casos los finales son $/ \mathrm{L}^{*}+\mathrm{H} \mathrm{H} \% /$. Por tanto, tampoco coinciden nuestros resultados con los de Ávila (2003) que considera que el acento tonal predominante en el final de las interrogativas es $/ \mathrm{L}+\mathrm{H}^{*} \mathrm{H} \%$ / con la variante $\left[\mathrm{L}+\mathrm{H}^{*} \mathrm{H} \%\right]$ en finales llanos y $\left[\mathrm{H}^{*}\right.$ $\mathrm{H} \%$ ] en los agudos. Solo cuando la tónica queda baja se coloca en el mínimo de F0, por lo que no podemos extender esta afirmación a todos los casos (Sosa 1999; Congosto 2012). Finalmente, no hemos encontrado en nuestro análisis el final descendente ( $\left.\mathrm{L}^{*} \mathrm{~L} \%\right)$ descrito 
por Ávila (2003) y, respecto del final circunflejo mencionado por Quilis (1993), solo hemos registrado en los finales agudos de los hombres el acento tonal $/ \mathrm{L}+\mathrm{H}^{*} /$ seguido de un tono de frontera medio / $\mathrm{M} \% /$, pero este último solo se registró en un 4,2\% de casos.

\section{Conclusiones}

El presente trabajo se ha planteado como una aportación parcial al estudio de la entonación del español de San Antonio, Texas y, por tanto, las conclusiones no pretenden agotar el tema de estudio. Hemos encontrado similitudes importantes entre la entonación del espańol de los hablantes ingleses de San Antonio analizados y la mexicana como, por ejemplo, la gran subida que experimentan los finales de las interrogativas desde el valle tonal hasta el final. En otros estudios iremos comprobando si las semejanzas y las diferencias observadas respecto de la entonación mexicana se dan en corpus diferentes y en otros informantes de San Antonio para poder evaluar la importancia de las mismas en el marco de bilingüismo que se da en esta zona sureña de EE.UU. Por otra parte, al tener en cuenta el tono medio (TM) para delimitar los tonos de frontera inicial y final y un umbral diferencial $(1,5 \mathrm{St})$, se han podido fijar de manera coherente y sistemática las invariantes y variantes de ambos tonos. También ha resultado eficaz separar las oraciones según sus inicios (TA o ATA) puesto que hemos comprobado que el tono de frontera inicial alto o bajo varía, aunque no de manera sistemática, en función de un inicio u otro. Asimismo, se ha comprobado la eficacia de separar el estudio de las frases según sus finales puesto que se ha visto que, si bien en agudos y llanos se da un descenso en las declarativas y un ascenso en las interrogativas, el comportamiento tonal varía de un final a otro: en las declarativas se da un salto característico de la pretónica a la tónica en agudos, pero de la tónica a la postónica en los llanos. Por su parte, en los finales agudos de las interrogativas, salvo excepciones, la tónica queda alta, mientras que en los llanos queda baja, por lo que el etiquetaje fonéticofonológico no coincide con el que dan otros autores para las interrogativas mexicanas.

\section{Obras Citadas}

Anderson-Hsieh, Janet, Ruth Johnson y Kenneth Koehler. 1992. "The Relationship Between Native Speaker Judgments of Nonnative Pronunciation and Deviance in Segmentais, Prosody, and Syllable Structure". Language Learning 42: 529-555.

Ávila, Sylvia. 2003. "La entonación del enunciado interrogativo en el español de la ciudad de México". En Esther Herrera y Pedro Martín Butragueño, Eds., La tonía. Dimensiones fonéticas y fonológicas. México, DF: El Colegio de México. 331-355.

Congosto Martín, Yolanda. 2012. "Lenguas en contacto y prosodia. La entonación del español en hablantes bilingües mexicoamericanos". Lingüistica española actual 34.1.: 137-166. 
Dorta, Josefa (ed.). 2013. Estudio comparativo preliminar de la entonación de Canarias, Cuba y Venezuela. Madrid-Santa Cruz de Tenerife: La Página ediciones S/L, Colección Universidad.

Ennis, Sharon, Merarys Ríos-Vargas \& Nora Albert. 2010. The Hispanic Population: [en línea: http://www.census.gov/prod/cen2010/briefs/c2010br-04.pdf, 16/01/2017].

Escobar, Anna María y Wolfgang Wölck (eds.). 2009. Contacto lingüistico y la emergencia de variantes y variedades lingüisticas. Madrid/Frankfurt: Iberoamericana/Vervuert.

Kelm, Orlando. 1995. "Acoustic Measurements of Spanish and English Pitch Contours: Native and Non-Native Speakers". Hispanic Linguistics 6/7: 435-448.

Lacorte, Manel y Jennifer Leeman (eds.). 2009. Español en Estados Unidos y otros contextos de contacto. Sociolingüistica, ideología y pedagogía. Madrid/Frankfurt: Iberoamericana/ Vervuert.

Ladd, David Robert. 1996. Intonational Phnology. Cambridge: Cambridge University Press.

López Bobo, María Jesús et al. 2007. "Análisis y representación de la entonación. Replanteamiento metodológico en el marco del proyecto AMPER". En Dorta, Josefa, ed., La prosodia en el ámbito lingüistico románico. Santa Cruz de Tenerife: La Página ediciones S/L, Colección Universidad. 17-34.

Manzanares Rivera, José Luis. 2015. "Los México-americanos en San Antonio, Texas: Análisis de su inserción en autoempleo formal". Estudios Fronterizos 16.31: 175-205.

Marcos Marín, Francisco Antonio. 2014. "Para la etnolingüística del español de San Antonio, Tejas". Revista Iberoamericana de Lingüistica, 9: 97-132. 2006. Los retos del español. Madrid: Iberoamericana/Vervuert.

Martín Butragueño, Pedro. 2006. "El estudio de la entonación del español de México". En Mercedes, Sedano, Adriana Bolívar y Martha Shiro, eds., Haciendo lingüistica. Homenaje a Paola Bentivoglio. Caracas: Universidad Central de Venezuela. 105-126. . 2004. "Configuraciones circunflejas en la entonación del español mexicano". Revista de Filología Española 84, 2: 347-373.

Matluck, Joseph H. 1965. "Entonación hispánica”. Anuario de Letras 5: 5-32.

Moreno Fernández, Francisco. 2008. "Dialectología hispánica en los Estados Unidos”. En Humberto López Morales, coord., Enciclopedia del español en Estados Unidos. Anuario del Instituto Cervantes. 200-221.

Mota, Carme de, Pedro Martín Butragueño \& Pilar Prieto. 2010. "Mexican Spanish Intonation, en Pilar Prieto \& Paolo Roseano, Eds., Transcription of Intonation of the Spanish Language. München: Lincom Europa. 319-350.

Pamies Bertrán, Antonio, Ana María Fernández Planas, Eugenio Martínez Celdrán, Alicia Ortega y Ma. Cruz Amorós. 2002. "Umbrales tonales en el español peninsular". Actas del II Congreso de Fonética Experimental. Sevilla: Universidad de Sevilla. 272-278.

Boersma, Paul y David Weenink. 2016. Praat, versión 6.0.19.

Quilis, Antonio. 1993. Tratado de fonética y fonología españolas. Madrid: Gredos. 190. . 1985. "Entonación dialectal hispánica”. Lingüistica Española Actual VII: 145- 
Rietveld, Toni y Carlos Gussenhoven. 1985. "On the relation between pitch excursion size and pitch prominence". Journal of Phonetics 13: 299-308.

Silva Corvalán, Carmen. 1996. Language contact and change: Spanish in Los Angeles. Oxford: Clarendon.

Sosa, Juan Manuel. 1999. La entonación del español. Su estructura fónica, variabilidad y dialectología. Madrid: Cátedra.

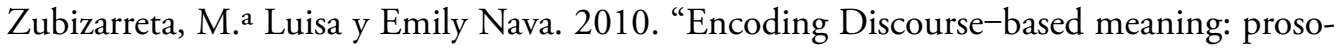
dy vs. syntax. Implications for Second Language acquisition". Lingua 121, Issue 4: 652-669. 
